\title{
Compound Sets in Mathematical Programming Modeling Languages
}

\author{
J. J. Bisschop • C. A. C. Kuip \\ University of Twente, Department of Applied Mathematics, P.O. Box 217, 7500 AE Enschede, The Netherlands
}

$\mathrm{T}$ his paper presents a series of simple but realistic examples in which common algebraic indexing conventions are not so convenient. In particular, it analyzes the difficulties caused by the conventions that each model component must have a fixed number of indices and that the order of the indices is significant to their meaning. To deal with these difficulties compensating extensions to algebraic notation are proposed. The proposed notation is compared to existing notation in terms of the human abilities to understand, maintain and verify model descriptions. (Mathematical Programming; Modeling Language; Index Sets)

\section{Introduction}

\subsection{Scope and Purpose}

Many designs for mathematical programming software have incorporated the idea of an algebraic modeling language. Such a language employs the conventions of common algebraic notation to describe objectives and constraints in a form that computers can process. The modeling language approach has the advantage of using a form that has great expressive power, and yet that is already familiar to many users.

Whatever its advantages, however, algebraic notation did not originally evolve with mathematical programming applications in mind. Certain kinds of large and complex models are thus more-or-less awkward to express within the standard algebraic conventions. To the extent that modeling languages adopt these conventions, they can also be awkward to use.

Some especially problematical aspects of algebraic notation arise in the indexing of collections of model components. In a simple model one can define, say, the indexing sets $I$ of factories and $J$ of warehouses; then it is possible to define a capacity $a_{i}$ at each factory $i \in I$, a variable $x_{i, j}$ representing the amount shipped from each factory $i \in I$ to each warehouse $j \in J$, and so forth. Finally, one can write $\sum_{j \in l} x_{i, j}$ for the total shipments out of a factory $i \in I$, and can define an indexed collection of constraints such as $\sum_{j \in J} x_{i, j} \leq a_{i}$ for each $i \in I$.
All of this can be carried over very nicely into a modeling language, as the designs of GAMS (Brooke et al. 1988) and AMPL (Fourer et al. 1990) have shown.

This paper presents a series of simple but realistic examples in which algebraic indexing conventions are not so convenient. In particular, it analyzes the difficulties presented by two common algebraic conventions: first, that each model component must have a fixed number of indices (one for $a_{i}$, two for $x_{i, j}$, and so on); and second, that the order of indices is significant to their meaning $\left(x_{i, j}\right.$ is always the amount shipped from $i$ to $j$, for instance). To deal with these difficulties, several compensating extensions to algebraic notation are proposed.

These extensions serve to enhance the ability to understand, maintain and verify model descriptions, and carry over to modeling languages for computers just as well as the more traditional notation.

The material of this paper has the potential to be of interest to designers of future modeling languages, as well as to the greater audience of modelers whose needs will influence future designs. The paper may also have the broader virtue of encouraging close analysis of modeling language features that have previously been taken for granted.

This paper is one in a series of papers which treat modeling languages as a research topic. A first analysis is presented in Fourer (1983). Other papers discuss new 
languages (Anthonisse 1970, Ashford and Daniel 1987, Bisschop and Meeraus 1982, Hürlimann and Kohlas 1988, Lucas and Mitra 1988, Meeraus 1983, Sagi 1986, Simons 1987), new concepts (Bradley and Clemence 1987; Geoffrion 1987, 1989; Jones 1990), data management (Fourer 1991), model management (Dolk and Konsynski 1984, Palmer et al. 1984) and error and infeasibility checking (Bisschop 1988, Greenberg 1983).

\subsection{The Order and Meaning of Indices}

This subsection motivates the basis of compound sets as treated in this paper. It analyzes the difficulties presented by the two common algebraic conventions, namely that the order of indices is significant to their meaning and that each model component must have a fixed number of indices.

Consider the following two individual variables in a model description or output report where Transport refers to a level of transport:

$$
\begin{aligned}
& \text { Transport (DC, NY, Boston). } \\
& \text { Transport (Boston, NY, DC). }
\end{aligned}
$$

These variables are different, because the order of city names is significant to their meaning. Without further explanation it is not clear whether the first variable represents the level of transport from DC via NY to Bos ton or vice-versa.

The explanation needed is usually provided by a comment statement in the declaration of variables. Consider the following declaration of a variable as an illustration:

Transport $t_{i, k, j}$ level of transport

$$
\text { from city } i \text { via city } k \text { to city } j \text { (tons). }
$$

In this commentary the phrase "level of transport" explains the meaning of the variable Transport further. The indices $i, k$ and $j$ are elements of the set "city", and the unit of measurement is specified as "tons".

The remaining three words are the labels "from", "via" and "to" relating the meaning of the indices $i, k$ and $j$ with the meaning of the variable Transport.

If such labels were to be an explicit part of mathematical notation, then the order of the city names is no longer significant to their meaning. The first variable in the above examples can be rewritten using the labels "from", "via" and "to" in the following ways:

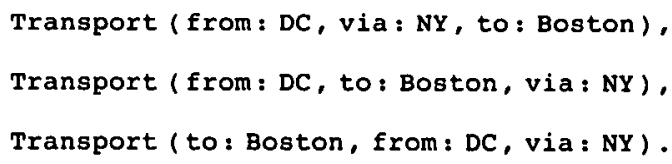

An extra benefit of using labels as an explicit part of notation is that there is no longer a requirement to have a constant number of indices for the same class of variables. Consider the following two different individual variables that are also taken from the above class of variables:

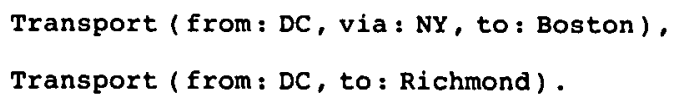

This is one example in which a single group of similar variables defined over several indices contains particular members for which selected indices are irrelevant. Another example is a group of variables representing production levels distinguished by product and quality in which quality is not relevant for every product. The reader may have encountered other such examples in which a group of similar variables does not have a uniform reference structure.

In this paper a proposal is made to incorporate labeled indices into the algebraic notation for the description of mathematical programming models.

\subsection{Outline}

A characterization of labeled compound sets is provided in $\S 2$. This section is a formal basis for the language constructs proposed in subsequent sections.

The concept compound set as it is currently supported by the languages AMPL and GAMS is reviewed in $\S 3$. This review is followed by a proposal to unify and extend the notion of compound set using the definitions of the previous section. An example is used to illustrate the differences in notation.

In $\S 4$ an example is presented to illustrate the use of nested tuples and lists within tuples based on the framework of compound sets as defined in this paper.

A discussion is presented in $\S 5$ on (1) linking labeled compound sets to data bases, (2) contrasting the conceptual and algebraic forms of a model, and (3) evaluating the influence of labeled compound sets on the human abilities to understand, maintain and verify model descriptions. At the end of this section several conclusions are made. 
In the appendix selected examples are rewritten using an extended compound set notation for the modeling languages AMPL and GAMS.

\section{Characterization of Compound Sets}

In this section the terminology of the paper will be defined and related to existing terminology.

A set is a collection of particular entities. These entities are the elements in the set. The notation $x \in X$ denotes that $x$ is an element of $X$. In the case that the entities cannot be decomposed, they are called atomic entities or atoms for short.

If $X_{1}, \ldots, X_{n}$ are nonempty sets, then the cartesian product $X_{1} \times \cdots \times X_{n}$ is a set itself, and is defined as the collection of all ordered tuples $\left(x_{1}, \ldots, x_{n}\right)$ where $x_{i}$ $\in X_{i}$. Each item $x_{i}$ in the tuple $\left(x_{1}, \ldots, x_{n}\right)$ is called a component. Identical components are permitted. In that case they can only be distinguished on the basis of their position in the tuple.

A compound set is a subset of a cartesian product. This definition corresponds to the terminology used in Fourer et al. (1990).

A label is a string of characters to uniquely identify a component in a tuple. If a component $x_{i} \in X_{i}$ is assigned label $l_{i}$, then this labeled component is written as $l_{i}: x_{i}$ and is an element of $l_{i}: X_{i}$.

If $X_{1}, \ldots, X_{n}$ are nonempty sets and $l_{1}, \ldots, l_{n}$ are unique labels, then the labeled cartesian product $l_{1}: X_{1}$ $\times \cdots \times l_{n}: X_{n}$ is defined as the collection of all labeled tuples $\left(l_{i_{1}}: x_{i_{1}}, \ldots, l_{i_{m}}: x_{i_{m}}\right)$, where $\left\{i_{1}, \ldots, i_{m}\right\} \subseteq\{1$, $\ldots, n\}, x_{i_{j}} \in X_{i_{j}}, l_{i_{j}} \neq l_{i_{k}} \forall j \neq k$, and $1 \leq m \leq n$. Note that the length of labeled tuples is not constant, and that the position of each labeled component is not fixed. Two labeled tuples which differ only in the ordering of their labeled components are considered to be identical.

A labeled compound set is a subset of a labeled cartesian product.

An index is an identifier that refers to an element in a set. Such a set is referred to as an index set. The term compound index is used to indicate that the underlying index set is a compound set. For example, in the specification of a collection of availability constraints: $\forall i$ $\in I: \sum_{j \in I} x_{i, j} \leq a_{i}$ the identifiers " $i$ " and " $j$ " are indices.
One or more of the nonempty sets $X_{i}$ in a (labeled) cartesian product may be (labeled) cartesian products themselves. In that case the cartesian product is said to be nested, and its subsets are called nested compound sets.

The collection of labels in a particular tuple $t$ $=\left(l_{1}: x_{1}, \ldots, l_{m}: x_{m}\right)$ in a labeled compound set is $\left\{l_{1}, \ldots, l_{m}\right\}$, and is denoted by the set-valued function labels $(t)$.

The selection of a particular component in a labeled tuple can be viewed as a projection over the labeled components. The label itself can be used to represent the projection operator as follows. Consider a labeled tuple $t=\left(l_{i_{1}}: x_{i_{1}}, \ldots, l_{i_{m}}: x_{i_{m}}\right)$ in the labeled compound set $T=l_{1}: X_{1} \times \cdots \times l_{n}: X_{n}$. The projection is then written as

$$
l_{i}(t)= \begin{cases}x_{i} & \text { if } l_{i}: x_{i} \text { is a component in } t \\ \text { nonexistent } & \text { otherwise. }\end{cases}
$$

Tuples may be compared to test whether one tuple is contained in another tuple, analogous to testing whether one set is a subset of another. A labeled tuple $t_{1}$ is part_of labeled tuple $t_{2}$ whenever each labeled component of $t_{1}$ is also a labeled component of $t_{2}$. This is written as $t_{1} \subseteq t_{2}$. The part_of operator $\sqsubseteq$ is a logical operator resulting in either true or false (just as the $\subseteq$ operator).

A list is an ordered nonempty finite sequence of elements belonging to a single nonempty set. If $x_{1}, \ldots, x_{n}$ $\in X$, then $\left[x_{1}, \ldots, x_{n}\right]$ is a list. Note that in a list, unlike in a set, an element $x_{i}$ may be identical to an element $x_{j}$. The set of lists that can be formed with elements of a set $X$ is denoted by List_of $(X)$. The set List_of $(X)$ contains infinitely many elements. For instance, if $X=\{a\}$ then

$$
\text { List_of }(X)=\{[a],[a, a],[a, a, a], \cdots\} \text {. }
$$

This set-valued function is only part of domain specifications of compound sets. It is therefore not necessary to enumerate all elements of List_of $(X)$, but only to check whether a particular list $\left[x_{1}, \ldots, x_{n}\right]$ is an element of List_of $(X)$, i.e., to check whether $x_{1} \in X$ and . . . $x_{n} \in X$.

The concepts defined in this section will be illustrated via the examples in the subsequent sections of this paper. 


\section{Compound Sets in Algebraic Notation}

In this section a detailed example is used to illuminate the differences between common algebraic notation, the use of compound sets in AMPL and GAMS, and a proposed use of labeled compound sets.

\subsection{Common Algebraic Notation}

The following portion of an extended transportation model, stated in common algebraic notation, incorporates six indices and consists of an objective function, an availability constraint, a demand constraint, and a capacity constraint. The capacity constraint limits the throughput per transshipment point for each product and time period. A more fully-developed version of this model may also contain multicommodity constraints to tie the products together, inventory constraints to tie the periods together, and upper limits on the transport per mode.

\section{Sets}

$C$

$P$

$T$

$M$

It $\subseteq \mathrm{C} \times \mathrm{C} \times \mathrm{C} \times \mathrm{P} \times \mathrm{T} \times \mathrm{M}$ Itineraries,

$\begin{array}{lrrl}A \subseteq C \times & P \times T & \text { Sources, } \\ D \subseteq & C \times P \times T & \text { Sinks, } \\ L \subseteq & C \times & P \times T & \text { Links. }\end{array}$

City names,

Products

Time periods,

Modes of transport,

\section{Parameters}

Cost $_{i, k, j, p, t, m}$-Variable cost from city $i$ via city $k$ to city $j$ of product $p$ during period $t$ using transport mode $m$; $i, k, j \in C, p \in P, t \in T, m \in M$.

$A v_{i, p, t}-$ Availability at city $i$ of product $p$ during period $t ; i \in C, p \in P, t \in T$.

$\operatorname{Dem}_{j, p, t}-$ Demand at city $j$ of product $p$ during period $t ; j \in C, p \in P, t \in T$.

Cap $_{k, p, 1}$-Transport capacity of product $p$ via city $k$ during period $t ; k \in C, p \in P, t \in T$.

\section{Variables}

$X_{i, k, j, p, t, m}-$ Transport level from city $i$ via city $k$ to city $j$ of product $p$ during period $t$ using transport mode $m$; $i, k, j \in C, p \in P, t \in T, m \in M, X_{i, k, j, t, t, m} \geq 0$.

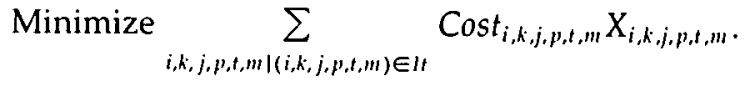

Availability Constraint

$\forall i, p, t \mid(i, p, t) \in A: \sum_{k, j, m \mid(i, k, j, p, t, m) \in I t} X_{i, k, j, p, t, m} \leq A v_{i, p, t}$.

Demand Constraint

$\forall j, p, t \mid(j, p, t) \in D$ :

$$
\sum_{i, k, m \mid(i, k, j, p, t, m) \in H} X_{i, k, j, p, t, m} \leq D e m_{j, p, t} .
$$

\section{Capacity Constraint}

$\forall k, p, t \mid(k, p, t) \in L:$

$$
\sum_{i, j, m \mid(i, k, j, p, t, m) \in I t} X_{i, k, j, p, t, m} \leq \operatorname{Cap}_{k, p, t} .
$$

In this example using common algebraic notation the compound sets $I t, A, D, L$ are used to select appropriate tuples from a cartesian product. In this way the elements in compound sets are referenced indirectly rather than directly. In the next subsections the above notation is adjusted to permit a direct referencing of the elements in compound sets.

\subsection{Compound Sets in AMPL and GAMS}

In AMPL it is possible to directly declare that a parameter or variable is defined for all elements in a compound set. Consider the following declaration of the parameter Dem and the variable $X$ based on the sets declared in the previous subsection.

$\operatorname{Dem}\{D\}$-Demand at sink, defined over $D$,

$X\{I t\}$-Transport level, defined over It .

In AMPL it is also possible to reference the elements in a compound set more directly than in common algebraic notation. This is illustrated next using a variation of algebraic notation that has the property of being directly representable in AMPL:

$$
\text { Minimize } \sum_{(i, k, j, p, t, m) \in I t} \operatorname{Cost}_{i, k, j, p, t, m} X_{i, k, j, p, t, m} .
$$

Availability Constraint

$$
\forall(i, p, t) \in A: \sum_{(i, k, j, p, t, m) \in l t} X_{i, k, j, p, t, m} \leq A v_{i, p, t} .
$$

Demand Constraint

$$
\forall(j, p, t) \in D: \sum_{(i, k, j, p, t, m) \in \| t} X_{i, k, j, p, t, m} \geq D e m_{j, p, t} .
$$




\section{Capacity Constraint}

$$
\forall(k, p, t) \in L: \sum_{(i, k, j, p, t, m) \in I t} X_{i, k, j, p, t, m} \leq \operatorname{Cap}_{k, p, t} .
$$

This notation is more concise but still lacks the facility to reference the elements of a compound set directly via a single compound index.

In GAMS 2.25 it is possible to use compound indices in the objective and constraint definitions. Compound sets, however, cannot be used in the declarations of variables as in AMPL. The use of compound indices in GAMS is illustrated next, again using a variation of algebraic notation that has the property of being directly representable in GAMS:

$$
\text { Minimize } \sum_{t t} \operatorname{Cost}_{t t} X_{l t} \text {. }
$$

\section{Availability Constraint}

$$
\forall a \in A: \sum_{(i, k, j, p, t, m) \in I t \mid(i, p, t)=a} X_{I t} \leq A v_{a} .
$$

Demand Constraint

$$
\forall d \in D: \sum_{(i, k, d, m) \in \| t} X_{\| r} \geq D e m_{d} .
$$

\section{Capacity Constraint}

$$
\forall l \in L: \sum_{(i, k, j, p, t, m) \in l l \mid(k, p, t)=1} X_{I t} \leq \mathrm{Cap}_{l} .
$$

The notation for the demand constraint is different from the notation for the availability and capacity constraints. In the demand constraint the compound index $d$ replaces the consecutive atomic indices $j, p$ and $t$ in the compound set $I t$. This results in a concise notation. In the availability constraint, however, the compound index $a$ cannot replace the atomic indices $i, p$ and $t$, because they are not consecutive indices in the compound set It .

Both the demand and the availability constraints can be written concisely if the atomic indices in the compound set $I t$ are reordered from $(i, k, j, p, t, m)$ to $(i, p, t, j, k, m)$ and the indices in the compound set $D$ are reordered from $(j, p, t)$ to $(p, t, j)$. It is straightforward to verify, however, that there is no single order of the indices that permits the use of compound indices in all three constraints stated in Example 3.1.

Despite the limitation imposed by index order there are clear advantages to the notations offered by AMPL and GAMS. One advantage is that whole portions of a model can be written in a concise manner. Another advantage is that the inherent sparsity of a compound set (being a subset of a cartesian product) is automatically incorporated into the model formulation.

The example in this subsection illustrates that compound indices cannot be fully employed in a notation that is based on a fixed order of indices. This limitation is removed in the next subsection in which labeled compound indices are introduced.

\subsection{Labeled Compound Sets}

The example of the previous subsection will now be rewritten with the use of labels and the part_of operator as introduced in Subsection 1.2 and $\S 2$.

Declarations with Labels.

$\begin{array}{ll}\text { Sets } \\ C & \text { City names, } \\ P & \text { Products, } \\ T & \text { Time periods, } \\ M & \text { Modes of transport, } \\ \text { It } & \text { Itineraries, } \\ A & \text { Sources, } \\ D & \text { Sinks, } \\ L & \text { Links. }\end{array}$

It $\subseteq$ (from : $C \times$ via : $C \times$ to : $C \times$

$A \subseteq$ (from : $C \times$ of : $P \times$ during : $T \times$ using $: M)$,

$D \subseteq($ of : $P \times$ during $: T \quad$,

$L \subseteq($ to : $C \times$ of : $P \times$ during $: T \quad$, via : $C \times$ of : $P \times$ during $: T$

\section{Parameters}

Cost $_{i}$-Variable cost over itinerary $i ; i \in I$, $A v_{a}$-Availability at source $a ; a \in A$, $\mathrm{Dem}_{d}$-Demand at sink $d ; d \in D$, Cap ${ }_{l}$-Transport capacity over link $l ; l \in L$. Variables

$X_{i}$-Transport level over itinerary $i ; i \in I t$.

$$
\text { Minimize } \sum_{i \in l t} \operatorname{Cost}_{i} X_{i} \text {. }
$$


Availability Constraint

$$
\forall a \in A: \sum_{i \in I \mid a \subseteq i} X_{i} \leq A v_{a} .
$$

Demand Constraint

$$
\forall d \in D: \sum_{i \in l|| \pi \kappa_{i}} X_{i} \geq \operatorname{Dem}_{d}
$$

\section{Capacity Constraint}

$$
\forall l \in L: \sum_{i \in I t \mid \mathbb{K}_{i}} X_{i} \leq \mathrm{Cap}_{l}
$$

The compound set notation with labels shares with AMPL and GAMS the conciseness obtained by the use of compound declarations and compound indices. The notation with labels, however, does not suffer from any limitations imposed by index order.

This concise notation is strongly dependent on the use of labels. With labels the component order of compound indices within one expression can be arbitrary, and the comparison between the tuples is straightforward. A further use of labels is emphasized in the next section.

\section{Nested Compound Sets with Lists}

In this section a different and more intricate example is introduced to demonstrate (1) the use of nesting in labeled compound sets, (2) the use of tuples with a varying number of components within a single labeled compound set, (3) the use of the function labels to verify domain requirements in tuples and (4) the use of lists for labeled components that all share the same label. The reader is referred to $\xi 2$ for the corresponding definitions.

\subsection{The Example and an Initial Analysis}

The following description of a transshipment problem for intermediate products serves as an example for the entire section:

Both an origin and a destination are described as a country and / or state coupled with the name of a factory. Each intermediate point is described as a country and / or state coupled with either the name of an airport or harbor. There is a set of viable transport routes from origins, possibly via a sequence of intermediate points, to destinations. Shipping cost per unit of product de- pends on the particular transport route (the total cost along a route is not necessarily equal to the sum of the costs for each connection along the route). Assume for the sake of the example that there is a demand constraint for each destination, and that there is a limit on the input capacity for each intermediate point. The question is how to model these two constraints and their corresponding data.

An initial analysis of the above problem statement provides the following suggestions.

- There are three collections of locations, namely origins, destinations and intermediate points. Each of these collections contains tuples with a variable number of components. For instance, either one or both of the components "country" and "state" are present. This suggests the use of labels to indicate which components are present.

- A route always contains an origin and a destination, but may or may not contain intermediate points. This suggests again the use of labels.

- The number of intermediate locations in a transport route is not determined beforehand. This suggests the use of a list to represent the sequence of intermediate points within a transport route.

- Transport routes consist of locations, and locations consist of other components. This suggests the use of nesting to represent routes.

On the basis of these suggestions the sets to be used for the demand constraint and the input capacity constraint are presented.

\subsection{The Specification of Sets}

$\begin{array}{lll}\text { Sets } & \text { Description } & \text { Abbreviated label } \\ C & \text { Country names } & \text { (cy) } \\ S & \text { State names } & \text { (st) } \\ F & \text { Factory names } & \text { (fac) } \\ A & \text { Airport names } & \text { (air) } \\ H & \text { Harbor names } & \text { (har) } \\ P & \text { Plant locations } & \\ T & \text { Transshipment points } & \\ & & \\ & P \subseteq(\text { cy }: C \times \text { st }: S) \times \text { fac }: F), \\ & T \subseteq(\text { cy }: C \times \text { st }: S) \times \text { har }: H \times \text { airport }: A) .\end{array}$

The labeled compound sets $P$ and $T$ contain tuples with varying number of components as illustrated below: 


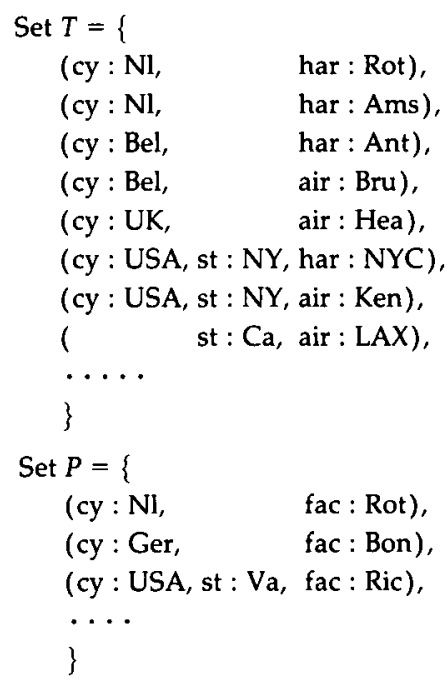

Once the labeled compound sets of locations have been declared, it is possible to declare the set of viable routes $R t$. In this declaration the concept of a list is used, see $\S 2$.

Set

$$
R t \subseteq(\text { from : } P \times \text { via : list_of }(T) \times \text { to }: P)
$$

Note that the declaration of $R t$ is independent of the declarations of $P$ and $T$. In this example the sets $P$ and $T$ are labeled compound sets, and $R t$ is thus a nested labeled compound set.

Part of the set $R t$ is shown next. Of the three route descriptions only the first two contain a list of intermediate locations:

$$
\begin{aligned}
& \text { Set } R t=\{ \\
& \text { (from : (cy: Nl, fac : Rot), } \\
& \text { via }:[(c y: \mathrm{Nl}, \quad \text { air }: \text { Sch }) \text {, } \\
& \text { (cy: UK, air : Hea )], } \\
& \text { to : (cy: USA, st : NY, air : Ken )), } \\
& \text { (from : (cy: Nl, fac : Rot), } \\
& \text { via }:[(c y: \mathrm{Nl}, \quad \text { air }: \text { Ams })] \text {, } \\
& \text { to : (cy:Bel, air : Bru )), } \\
& \text { (from : (cy : USA, st : NY, air : Ken), } \\
& \text { to : (cy: USA, st: Va, air : Ric )), } \\
& \ldots \ldots \\
& \text { \} }
\end{aligned}
$$

\subsection{Domain Specification and Verification}

Automatic verification whether tuples of a compound set are consistent with their domain specification is referred to as domain checking. It is one of the facilities offered by modeling languages such as GAMS and AMPL. For each tuple $t=\left(x_{1}, \ldots, x_{n}\right)$ in the compound set $T \subseteq X_{1} \times \cdots \times X_{n}$, it is checked whether $x_{i} \in X_{i}$ for each $i \in\{1, \ldots, n\}$. If $x_{i} \notin X_{i}$ for at least one $i$ $\in\{1, \ldots, n\}$, then this is called a domain violation.

The extension to labeled compound sets is straightforward. For each tuple $\left(l_{i_{1}}: x_{i_{1}}, \ldots, l_{i_{m}}: x_{i_{m}}\right)$ in a labeled compound set $T \subseteq l_{1}: X_{1} \times \cdots \times l_{n}: X_{n}$ it is checked that $l_{i_{j}}: x_{i_{j}} \in l_{i_{j}}: X_{i_{j}}$ for each $j \in\{1, \ldots, m\}$.

For labeled compound sets it is possible to provide extra facilities to verify whether tuples are consistent with required combinations of components. Consider the following requirements for the example in this section.

1. A transshipment point must contain at least one of the components labeled "cy" and "st".

2. A transshipment point must contain precisely one of the components labeled "har" and "air".

3. A route must at least contain the components labeled "from" and "to".

By using the set-valued function labels, as defined in $\S 2$, these three requirements can be verified as follows.

$$
\begin{aligned}
& \forall t \in T: \operatorname{card}(\text { labels }(t) \cap\{\text { cy, st }\}) \quad \geq 1, \\
& \forall t \in T: \operatorname{card}(\text { labels }(t) \cap\{\text { har, air }\})=1, \\
& \forall r \in R t: \operatorname{card}(\text { labels }(r) \cap\{\text { from, to }\})=2 .
\end{aligned}
$$

The formulation of these requirements in terms of unlabeled compound sets is not so concise. Such a formulation will require explicit references to an element "nonexistent" for particular positions in a tuple. It will also require an enumeration of permitted combinations of components in particular index positions.

\subsection{The Demand and Input Capacity Constraints}

The demand and input capacity constraints can be stated as follows:

Parameters

Dem $_{d}$-Demand at sink $d ; d \in D$,

Cap -Input capacity at throughpoint $t ; t \in T$.

Variables

$X_{r}$-Transport level over route $r ; r \in R t$.

Demand Constraint

$$
\forall d \in P: \sum_{r \in R t \mid \text { to }(r)=d} X_{r} \geq \operatorname{Dem}_{d} .
$$

Input Capacity Constraint

$$
\forall t \in T: \sum_{r \in R \mid t \in \operatorname{via}(r)} X_{r} \leq \text { Cap }_{t} .
$$


Note the use of labels as projection functions, as defined in $\S 2$. In the demand constraint the projection operator named "to" selects the component labeled "to" from a tuple in the labeled compound set $R t$. Whenever the demand point $d$ is equal to this component then the variable $X_{r}$ becomes a term in the summation.

In the input capacity constraint the projection operator "via" is used in a similar manner. This projection operator selects a list of intermediate points. Whenever the intermediate point $t$ is an element of this list, then the variable $X_{r}$ becomes a term in the summation. In the event that a route $r$ does not contain a "via" component, then $t \in \operatorname{via}(r)$ is false.

The above demand constraint can be rewritten using the part_of operator instead of the projection operator as follows:

\section{Demand Constraint}

$$
\forall d \in P: \sum_{r \in R(\mid+\circ: d) \in r} X_{r} \geq \operatorname{Dem}_{d} .
$$

A similar rewrite of the above input capacity constraint with the use of the part_of operator is not possible. The projection operator named "via" selects the component labeled "via" as a list. That is why it can be tested whether $t$ is, a component in the list. The part_of operator, however, is a boolean operator that can only compare two tuples and that cannot select a component from a list.

This example illustrates that both the projection operator and the part_of operator play a role in the use of labeled compound sets.

\section{Discussion and Conclusions}

\subsection{Labeled Compound Sets and Data Bases}

In relational databases, (see, e.g., Date 1986, Elmasri and Navathe 1989) the names of attributes (also referred to as columns) in tables can be used as the labels in labeled compound sets. In that case, linking a table in a relational database and a parameter or variable defined over a labeled compound sèt is, at least conceptually, straightforward.

Consider the example of $\S 3$ in which the parameter Cost and the variable $X$, both defined over the labeled compound set $I t$ of itineraries, are to be linked with a relational table, say ItinData.
The names of the key attributes in the table ItinData are from, via, to, of, during and using. The names of the derived attributes in table ItinData are, e.g., $x$ and cost. Facilities are provided in relational database languages such as SQL to update or retrieve the elements of the columns $X$ and cost.

The data of the example in $\S 4$ are not so easily linked to the standard relational data bases. One possibility might be the link to semantic databases (see, e.g., Hammer and McLeod 1981, Smith and Smith 1977). Both the framework and notation to be used for linking modeling languages to relational and semantic databases is still an important research topic (see, e.g., Fourer 1991). Consider, as an example, the correspondence between selected concepts in this paper and selected concepts in SDM (Hammer and McLeod 1981). This correspondence is summarized in Table 1 , and indicates a conceptual link between the data of the example in $\S 4$ and SDM.

\subsection{The Conceptual and Algebraic Forms of a Model}

A distinction can be made between a conceptual form of a model and an algebraic form of a model. A conceptual form is one in which a model is perceived in terms of entities and relationships between these entities without any restriction on notation. Such a form is subsequently translated into a formal algebraic notation describing the perceived entities and relationships.

The translation from a conceptual form of a model into its algebraic form is necessary for the implementation of that model on a computer. That is why it is of interest to study the differences between these two forms, so that future extensions to algebraic notation reduce the gap between them.

A first difference between a conceptual form of a model and its algebraic form is the extent in which the meaning of entities and relationships is explicitly stated. For instance, the "transport of goods from cities to cities" in a conceptual form is more meaningful than its formal algebraic counterpart "transport(goods, cities, cities)".

A second difference between a conceptual form of a model and its algebraic form is flexibility. For instance, in a conceptual form it does not matter whether the description of a route contains zero, one or more transshipment points. In the common algebraic description 
of such a route there is likely to be a separate index for each point along the route.

The proposal in this paper is to introduce the notion of labels into the formal algebraic notation for indexed variables. With this notion the two differences stated above between the conceptual form of a model and its algebraic form are reduced. For instance, with the use of labels "transport of goods from cities to cities" can be transcribed into "transport(of: goods, from: cities, to: cities)". Similarly, with the notion of labeled tuples and lists as proposed in this paper, routes can be described with a varying number of transshipment points.

\subsection{Understandability, Maintainability and Verifiability}

There are three important criteria to evaluate proposed extensions to modeling languages. They are related to the human abilities to understand, maintain and verify model descriptions. In general, these three abilities are strongly dependent on the communicative skills of the model builder, the complexity of the subject, and on the particular notation used. Next, only the influence of the particular mathematical notation is considered while applying these three criteria.

The understandability of a model can be enhanced with the introduction of labels. First, it has been illustrated in Subsection 1.2 that labels can be used to eliminate any ambiguity between components in a single tuple that are elements of the same set. In addition, the use of labeled compound sets permits a concise and clear notation of constraints as demonstrated in $\S \S 3$ and 4 .

Table 1 Relation between Concepts in This Paper and in SDM

\begin{tabular}{ll}
\hline & Concepts \\
\hline \multicolumn{1}{c}{ In this paper } & \multicolumn{1}{c}{ SDM } \\
\hline Set & Class \\
Label & Member attribute \\
Component & Value of attribute \\
Atom & String or number \\
Compound component & Value is entity in a class \\
List of components & Multivalued attributes \\
Domain & Value classes \\
\hline
\end{tabular}

The maintainability of a model can also be enhanced with the introduction of labels. Consider the example of $\S 3$. Removing the component "using" from the labeled compound set It does not affect any of the three constraints stated in Example 3.5.

Consider also the example of $\S 4$. A change of the labeled compound sets $P$ and $T$ to atomic index sets will again not affect the demand constraint and the input capacity constraint. Note that the reverse is also true. Consider a set of individual names whereby each name contains extra information and that this information is accessible via some name construction scheme. This information can now be made explicit by changing the individual names $x_{1}, \ldots, x_{n}$ to labeled tuples without affecting the constraints of the model.

The verifiability of a model can also be enhanced with the introduction of labels. Whenever two or more indices of a variable refer to elements from the same set, the consistency between the order of the indices and their meaning need no longer be verified (see, e.g., Subsection 1.2). In addition, as demonstrated in Subsection 4.3, domain checking with labels is enhanced by providing a facility for the verification of required combinations of components.

\subsection{Conclusions}

This paper indicates the potential of labeled compound sets to complement existing algebraic indexing conventions in mathematical programming models. While evaluating the proposed extensions in notation both critical and positive comments can be made.

A first critical comment is that the added facilities into the modeling language introduce new complexities that make the language more difficult to learn. A second critical comment is that the use of labels without the use of compound sets requires more keystrokes and makes a model less concise. A third critical comment is that the use of compound indices hides the individual atomic indices in assignments and equations.

Despite these criticisms this paper has clearly demonstrated that the introduction of both labels and compound sets offers several advantages. A first advantage is that the difficulties caused by the conventions that each model component must have a fixed number of indices and that the order of the indices is significant to their meaning are removed. A second advantage is 
that a model with many indices can be represented in a concise form. A third advantage is that a change in the number of components of a labeled compound set does not necessarily affect the structure of an equation written in terms of a such a compound set.

Making labels an explicit part of algebraic notation is new and unconventional. Whether or not their use will become common practice remains to be seen. It is hoped that this paper not only contributes to an ongoing evolution of modeling languages for mathematical programming, but that it also stimulates other researchers to suggest ways to improve the representation of mathematical programming models. ${ }^{1}$

1 The authors are grateful to the two anonymous referees who provided extensive commentary and detailed suggestions to improve both the original version and the first revision of this paper.

\section{Appendix}

This appendix illustrates how the concepts proposed in this paper might be incorporated into the existing modeling languages AMPL (Fourer et al. 1990) and GAMS (Meeraus 1983, Brooke et al. 1988). Selected examples presented in $\S \S 3$ and 4 are rewritten using the following extensions.

- Labels are used in the domain of sets, parameters and variables.

- The $\sqsubseteq$ operator (PARTOF in GAMS and partof in AMPL) is used in conditions relating tuples.

- The list_of operator (LISTOF in GAMS and listof in AMPL) is used in the declaration of components that are lists.

The examples are provided without a formal syntax.

Selected Examples in a Proposed Extension of AMPL

\section{EXAMPLE 3.5 .}

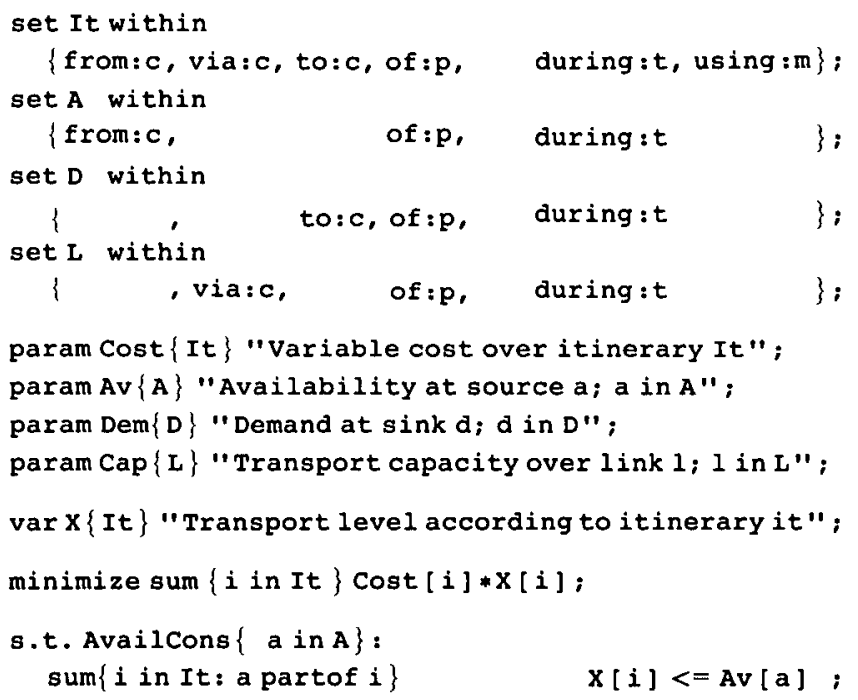

s.t. DemandCons $\{d$ in $D\}$ : $\operatorname{sum}\{i$ in $I t: d$ partof $i\}$

s.t. CapacCons $\{1$ in $\mathrm{L}\}$ : $\operatorname{sum}\{i$ in It: 1 partof $i\}$

$x[i]>=\operatorname{Dem}[d] ;$

$\mathrm{X}[\mathrm{i}]<=\operatorname{Cap}[1] ;$

EXAMPLE 4.1.

set $p$ within country:c, state:s, factory: $f$ \};

set $t$ within

$\{$ country:c, state:s, harbor:h, airport:a\};

EXAMPLE 4.3.

set Rt within from:p, via: listof $(t) *$ to: $p$ \};

EXAMPLE 4.6.

s.t. DemandCons $\{d$ in $P\}$ :

$\operatorname{sum}\{r$ in Rt: to $(x)=d\}$

s.t. Capaccons $\{t$ in $T\}$ :

$\operatorname{sum}\{r$ in $R t: t$ in via $(r)\}$

$X[r]>=\operatorname{Dem}[d]$

$X[r]<=\operatorname{Cap}[t] ;$

Selected Examples in a Proposed Extension of GAMS

EXAMPLE 3.5 .

SETS

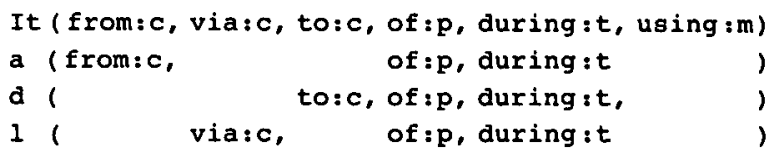

PARAMETERS

Cost (It) Variable cost over itinerary It

Av (a) Availability at source a

$\operatorname{Dem}(d)$ Demand at sink d

Cap (1) Transport capacity over link 1 ;

\section{VARIABLES}

$X$ (It) Transport level according to itinerary It obj Objective function value;

objective $\quad \cdots o b j=E=\operatorname{SuM}(I t, \operatorname{Cost}(I t) * X(I t))$; AvailCons (a) . .

$\operatorname{SUM}($ It $\$$ ( a PARTOF It ), $X(I t)) \quad=L=A v(a)$;

DemandCons (d) . .

sum( It $\$$ ( d PARTOF It ), $X(I t)) \quad=G=\operatorname{Dem}(d)$;

CapacCons (1) . .

SUM( It $\$(1$ PARTOF It ), $X(I t)) \quad=I=\operatorname{Cap}(1)$;

EXAMPLE 4.1.

SETS

P Plant locations

$t$ Transshipment points

p ( country:c, state:s, factory:f )

$t$ ( country:c, state:s, harbor:h, airport:a)

EXAMPLE 4.3.

SET Rt ( from:p, via:LISTOF ( $t$ ), to:p)

EXAMPLE 4.6 .

DemandCons (d) .

$\operatorname{SuM}(\operatorname{Rt} \$($ to $(R t) E Q d)), X(R t)) \quad=G=\operatorname{Dem}(d)$;

CapacCons $(t)$.

$\operatorname{SuM}(R t \$(t$ IN via(Rt)), X(Rt)) $\quad=L=\operatorname{Cap}(t)$; 


\section{References}

Anthonisse, J. M., "An Input System for Linear Programming Problems," Statistica Necrlandica, 24 (1970), 71-81.

Ashford, R. W. and R. C. Daniel, "LP-MODEL: XPRESS-LP's Model Builder," IMA I. of Mathematics in Management, 1 (1987), 163176.

Bisschop, J. J., "Language Requirements for A Priori Checking and Model Reduction in Large-scale Programming," In Mathematical Models for Decision Support, NATO ASI, Springer-Verlag, Berlin, 1988, 171-181.

— and A. Meeraus, "On the Development of a General Algebraic Modeling System in a Strategic Planning Environment," Mathematical Programming Study, 20 (1982), 1-29.

Bradley, G. H. and R. D. Clemence, Jr, "A Type Calculus for Executable Modeling Languages," IMA J. of Mathematics in Management, 1 (1987), 277-291.

Brooke, A., D. A. Kendrick and A. Meeraus, GAMS, A Users Guide, Scientific Press, Redwood City, CA, 1988.

Date, C. J., Database Systems, Vol. 1, fourth ed., Addison Wesley, Reading, MA, 1986.

Dolk, D. R. and B. R. Konsynski, "Knowledge Representation for Model Management Systems," IEEE Transactions on Software Engineering, 10 (1984), 619-628.

Elmasri, R. and S. B. Navathe, Fundamentals of Database Systems, Redwood City, CA, Benjamin / Cummings, 1989.

Fourer, R., "Modeling Languages Versus Matrix Generators for Linear Programming," ACM Transactions on Mathematical Software, 9 (1983), 143-183.

- "Database Structures for a Class of Mathematical Programming Models," Technical Report 90-06, Department of Industrial Engineering and Management Sciences, Northwestern University, Evanston, IL, January 1991.
- D. M. Gay and B. W. Kernighan, "A Modeling Language for Mathematical Programming," Management Sci., 36 (1990), 519554

Geoffrion, A. M., "An Introduction to Structured Modeling," Management Sci, 33 (1987), 547-588.

- "The Formal Aspects of Structured Modeling," Operations Res., 37, 1 (1989), 30-51.

Greenberg, H. J., "A Functional Description of ANALYZE: A Computer-assisted Analysis System for Linear Programming Models," ACM Transactions on Mathematical Software, 9 (1983) 18-56.

Hammer, M. and D. McLeod, "Database Description with SDM: A Semantic Database Model," ACM Transaction on Database Systems, 6,3 (1981), 351-386.

Hürlimann, T. and J. Kohlas, "LPL: A Structured Language for Linear Programming Modeling," OR Spektrum, 10 (1988), 55-63.

Jones, C. V., "An Introduction to Graph-based Modeling Systems, Part l: Overview," ORSA J. on Computing , 2, 2 (1990), 136-151.

Lucas, C. and G. Mitra, "Computer-assisted Mathematical Programming (Modeling) System: CAMPS," The Computer J, 31 (1988), $364-375$.

Meeraus, A., "An Algebraic Approach to Modeling," I. of Economic Dymanics and Control, 5 (1983), 81-108.

Palmer, K. H., N. K. Boudwin, H. A. Patton, A. J. Rowland, J. D. Sammes and D. M. Smith, A Model-management Framework for Mathematical Programming, An Exxon Monograph, Wiley, New York, 1984.

Sagi, I., "Computer-aided Modeling and Planning (CAMP)," $A C M$ Transactions on Mathematical Software, 12 (1986), 225-248.

Simons, R. V., "Mathematical Programming Modelling Using MGG," IMA J. of Mathematics in Management, 1 (1987), 267-276.

Smith, J. M. and D. C. P. Smith, "Database Abstractions: Aggregation," Conmunications of the ACM, 20, 6 (1977), 405-413.

Accepted by Thomas M. Liebling. Deparimental Editor; received July 1990. This paper has been with the authors 17 months for 2 revisions. 
Copyright 1993, by INFORMS, all rights reserved. Copyright of Management Science is the property of INFORMS: Institute for Operations Research and its content may not be copied or emailed to multiple sites or posted to a listserv without the copyright holder's express written permission. However, users may print, download, or email articles for individual use. 